\title{
Introduction to the Special Issue for SPAA 2016
}

\author{
SETH GILBERT, National University of Singapore
}

This special issue contains revised and extended versions of selected papers presented at the 28th ACM Symposium on Parallelism in Algorithms and Architectures (SPAA'16), held from July 11 to 13, 2016, at the Asilomar State Beach, California.

The goal of SPAA is to develop a deeper understanding of parallelism in all its forms, bringing together the theory and practice of parallel computing. Research at SPAA covers a wide diversity of topics under the umbrella of parallelism, broadly construed.

This special issue contains five papers from SPAA 2016 representing some of the best received papers from the conference. Following their invitation to the special issue, they have been rigorously peer reviewed following TOPC's standard review process. Overall, these articles well represent the diversity of parallel algorithms that are studied by the SPAA community, including traditional lock-free parallel data structures, resource allocation, distributed algorithms, parallel query models, and parallel information dissemination. These articles highlight the variety of exciting work in parallelism that was represented at SPAA 2016.

- The first article in this special issue is "Better Bounds for Coalescing-Branching Random Walks" by Michael Mitzenmacher, Rajmohan Rajaraman, and Scott Roche. This article studies a natural type of random walk known as a "cobra walk" that models the spread of disease in a population or the spread of information in networks. They study the cover time of cobra walks on $d$-regular and general graphs, showing (for example) that information is disseminated more quickly with cobra walks than with classical random walks. The results of this article provide powerful new tools for understanding and designing algorithms that rely on random walks and their variants.

- The second article is "Randomized Approximate Nearest Neighbor Search with Limited Adaptivity” by Mingmou Liu, Xiaoyin Pan, and Yitong Yin. This article looks at the problem of approximate nearest neighbor search in a $d$-dimensional Hamming space. They consider a parallel query model where the algorithm can make $k$ rounds of queries to the data structure. They develop two randomized algorithms that minimize the total number of memory accesses, along with a lower bound demonstrating where their algorithms are asymptotically optimal. Overall, these results nicely illustrate the benefits of parallelism via the tradeoff between time (in terms of rounds) and total cost (in terms of memory accesses).

- The third article is "Fast Distributed Algorithms for Connectivity and MST in Large Graphs" by Gopal Pandurangan, Peter Robinson, and Michele Scquizzato. This article looks at the problem in which a distributed collection of servers are cooperating to solve a graph problem, e.g., determining whether the input graph is connected or finding an MST. The article assumes that the input graph is initially randomly partitioned among the servers, and they

\footnotetext{
Author's address: S. Gilbert, National University of Singapore; email: seth.gilbert@comp.nus.edu.sg.

Permission to make digital or hard copies of part or all of this work for personal or classroom use is granted without fee provided that copies are not made or distributed for profit or commercial advantage and that copies bear this notice and the full citation on the first page. Copyrights for third-party components of this work must be honored. For all other uses, contact the Owner/Author.

2018 Copyright is held by the owner/author(s).

2329-4949/2018/07-ART1

https://doi.org/10.1145/3230677
} 
study how many rounds of communication are necessary to solve the problem. They give an (almost) optimal distributed randomized algorithm for graph connectivity, and leverage these techniques to solve a variety of other graph problems. All together, the results in this article make important progress on a fundamental distributed algorithms question, specifically, how to solve graph problems in a distributed setting.

- The fourth article is "Robust and Probabilistic Failure-Aware Placement" by Madhukar Korupolu and Rajmohan Rajaraman. This article looks at the problem of assigning tasks to fault-prone machines in a data center so as to ensure a certain amount of reliability. This is a challenging optimization problem, and they prove several results characterizing the complexity of the problem. And the article develops several new algorithms for this setting, including a PTAS for one variant and an approximation algorithm for a hierarchical variant. (An interesting aspect of these results is their notion of fairness, which generalizes traditional max-min fairness.) In total, this article contains a large collection of results that provide significant insight into a hard, but important, assignment problem.

- The fifth article is "Lock-free Transactional Transformation for Linked Data Structures" by Deli Zhang and Damian Dechev. This article shows how to transform high-performance lock-free data structures into transactional data structures where operations can be grouped into transactions that are executed in a linearizable all-or-nothing manner. The article focuses on linked data structures, applying their technique to linked lists and skiplists. They prove that their approach is correct, as well as giving an experimental evaluation of its performance. This article develops an important alternative to transactional memory for designing a data structure that supports transactions.

We would like to thank the many people who made this special issue possible, including the SPAA 2016 PC for helping to select and review these papers, along with the other reviewers who provided careful reviews and useful feedback. We would also like to thank Editor-in-Chief Phil Gibbons for organizing this special issue and the ACM publication staff for making the whole process run smoothly. 